\title{
Tensorial perturbations in the bulk of inflating brane worlds
}

\author{
Pedro F. González-Díaz \\ Centro de Física "Miguel A. Catalán," Instituto de Matemáticas y Física Fundamental, Consejo Superior de Investigaciones Científicas, \\ Serrano 121, 28006 Madrid, Spain \\ (Received 1 July 2003; published 23 October 2003)
}

\begin{abstract}
In this paper we consider the stability of some inflating brane-world models in quantum cosmology. It is shown that, whereas the singular model based on the construction of inflating branes from Euclidean fivedimensional anti-de Sitter space is unstable to tensorial cosmological perturbations in the bulk, the nonsingular model which uses a five-dimensional asymptotically anti-de Sitter wormhole to construct the inflating branes is stable to these perturbations.
\end{abstract}

DOI: 10.1103/PhysRevD.68.084009

PACS number(s): 04.25.Nx, 04.50.+h, 98.90.+s

\section{INTRODUCTION}

There has recently been a lot of interest in inflating brane worlds which are obtained by gluing at a given slice two copies of either a truncated anti-de Sitter or regular wormhole spacetime in five dimensions [1,2]. These brane worlds can in both cases evolve along cosmological time following the conventional pattern of most cosmic branes (i.e. the evolution being described by a Friedmann equation which shows an early dependence on the square of the energy density [3]) after undergoing a primordial period of pure de Sitter inflation. It is then clear that the four branes resulting in these models are by themselves stable to all types of cosmological perturbations taking place in the branes [4]. However, no investigation has yet been undertaken on the stability of the bulk under the same type of perturbations in the above two models. In this paper we shall study in some detail this important missing topic in the case in which the cosmological perturbations originally considered by Lifshitz and Khalatnikov [5] are extended to a five-dimensional manifold [6]. The main result of this study is that, whereas the construct obtained from the five-dimensional anti-de Sitter space is unstable to tensorial cosmological perturbations, the construct which is obtained from a regular five-dimensional asymptotically anti-de Sitter wormhole is stable to these perturbations.

We can outline the paper as follows. In Sec. II we discuss the instabilities present in the instanton constructed from the five-dimensional anti-de Sitter space. Section III contains a calculation which leads to the conclusion that, whereas the four-dimensional asymptotically anti-de Sitter shows tensorial instabilities, the five-dimensional asymptotically anti-de Sitter wormhole bulk of a four-dimensional brane is stable to tensorial perturbations. We conclude in Sec. IV.

\section{INSTABILITIES OF DE SITTER BRANE WORLDS}

The scale factor of the five-dimensional Garriga-Sasaki model for an inflating brane world, which describes a spacetime with topology $R \times S^{4}$, i.e., denoting the metric on the unit four-sphere by $d \Omega_{4}^{2}$,

$$
d s^{2}=d r^{2}+a(r)^{2} d \Omega_{4}^{2},
$$

is given by [1]

$$
a(r)=\ell \sinh (r / \ell),
$$

where $\ell=\left(-6 / \Lambda_{5}\right)^{1 / 2}$ is the anti-de Sitter radius and $r$ is the extra fifth coordinate. It was pointed out in Refs. [1,2] that this solution may lead to a problem. The problem is that the nonvanishing components of the five-dimensional Weyl tensor $C_{\nu \rho \sigma}^{\mu}$ give rise to the invariant quantity $C^{2} \propto a(r)^{-4}$ which may vanish at $r=0$ if we take for $a(r)$ solution (2.1). This shortcoming was circumvented by using the same spacetime topology as in the Garriga-Sasaki model, but for a scale factor given by

$$
a(r)=\left(\frac{\sqrt{\beta} \cosh \left(2 \sqrt{\Lambda_{5}} r\right)-1}{2 \Lambda}\right)^{1 / 2}
$$

(with $\beta=1+4 A^{2} \Lambda_{5}$ ) while still producing the same de Sitter inflating brane-world model.

In this paper it will be seen that the solution used by Garriga-Sasaki is unstable in yet another respect, that of the gravitational-wave perturbations on the bulk, and that using again a solution such as Eq. (2.2) also solves this new problem for constructing an inflating de Sitter brane-world scenario. This can be explicitly shown by considering the Lifshitz-Khalatnikov tensorial cosmological perturbations [5] generalized to a five-dimensional Friedmann-RobertsonWalker manifold [6] for the scale factors given by Eqs. (2.1) and (2.2). Expanding in five-dimensional tensor harmonics and taking for the most general metric perturbations [6]

$$
h_{\alpha \beta}=\lambda(\eta) P_{\alpha \beta}+\mu(\eta) Q_{\alpha \beta}+\sigma(\eta) S_{\alpha \beta}+\nu(\eta) H_{\alpha \beta},
$$

where $P_{\alpha \beta}, Q_{\alpha \beta}, S_{\alpha \beta}$, and $H_{\alpha \beta}$ are tensor harmonics derived from the scalar, vector, and tensor harmonics defined on the four-sphere [6], and the coefficients $\lambda(\eta), \mu(\eta)$, $\sigma(\eta)$, and $\nu(\eta)$ are functions of the conformal extra coordinate $\eta=\int d r / a(r)$. The perturbations originated from the tensorial five-dimensional gravitational-wave perturbations are given by coefficient $\nu(\eta)$ which satisfies a differential equation which in the Lorentzian manifold reads [6]

$$
\nu^{\prime \prime}+3 \frac{a(\eta)^{\prime}}{a(\eta)} \nu^{\prime}+\ell(\ell+3) \nu=0,
$$


with ${ }^{\prime}=d / d \eta$, where $\eta$ is again the conformal coordinate associated with the extra fifth dimension $r$, i.e., $\eta$ $=\int d r / a(r)$, with $a(r)$ given by Eq. (2.2) for the present case. What we shall investigate in this paper is how tensorial perturbations on the four-sphere $\Omega_{4}$, represented by the coefficient $\nu$, evolve along either the "Lorentzian" or the "Euclidean" conformal fifth coordinate for a generic metric

$$
d s^{2}=a(\eta)^{2}\left( \pm d \eta^{2}+d \Omega_{4}^{2}\right),
$$

with the upper sign standing for the Euclidean manifold and the lower one for the Lorentzian manifold.

In the Euclidean case, which corresponds to the metric used by Garriga and Sasaki [1], we have

$$
\nu^{\prime \prime}+3 \frac{a(\eta)^{\prime}}{a(\eta)} \nu^{\prime}=\ell(\ell+3) \nu,
$$

where

$$
\eta=\eta_{*}+\frac{1}{2}+\ln \left(\frac{\cosh \left(\sqrt{\Lambda_{5}} r\right)-1}{\cosh \left(\sqrt{\Lambda_{5}} r\right)+1}\right),
$$

in which $\eta_{*}$ is an integration constant. The scale factor expressed in terms of the conformal radial coordinate $\eta$ becomes then

$$
a(\eta)=\frac{1}{\sqrt{\Lambda_{5}} \sinh \left(\eta-\eta_{*}\right)} .
$$

The differential equation (2.4) can therefore be written as

$$
\nu^{\prime \prime}-3 \operatorname{coth}\left(\eta-\eta_{*}\right) \nu^{\prime}=\ell(\ell+3) \nu .
$$

We note that even for the zero mode $\ell=0$ there is an instability, as the solution to this equation reads

$$
\nu=\nu_{0}+\nu_{0}^{\prime}\left[\frac{1}{3} \cosh ^{3}\left(\eta-\eta_{*}\right)-\cosh \left(\eta-\eta_{*}\right)\right],
$$

in which $\nu_{0}$ and $\nu_{0}^{\prime}$ are integration constants. It follows that for $\eta \rightarrow \infty$ (i.e., as $r \rightarrow 0$ ) $\nu$ blows up. In the corresponding Lorentzian case this mode would oscillate along the timelike extra dimension. The conclusion thus obtained is that the five-dimensional bulk is unstable for solution (2.1) or (2.6). The stability of the generalized $d$-dimensional GarrigaSasaki scenario can also be analyzed following a similar treatment. Since the Garriga-Sasaki metric for an arbitrary number of dimensions $d$ is found to be tractable [7] and expressible as

$$
d s^{2}=a(\eta)^{2}\left( \pm d \eta^{2}+d \Omega_{d-1}^{2}\right)
$$

with $a(\eta)$ given again by Eq. (2.6) for a $d$-dimensional cosmological constant $\Lambda_{d}$, the differential equation for tensorial perturbations $\nu[6]$ for the Euclidean case becomes

$$
\nu^{\prime \prime}-(d-2) \operatorname{cotanh}\left(\eta-\eta_{*}\right) \nu^{\prime}=\ell(\ell+d-2) \nu .
$$

For $\ell=0$, we now obtain

$$
\nu^{\prime}=\nu_{0}^{\prime} \sinh ^{d-2}\left(\eta-\eta_{*}\right),
$$

with $\nu_{0}^{\prime}$ an integration constant. It follows that for $\ell=0$ the coefficient $\nu$ will be generally given as polynomials of certain powers, $p \leqslant d$, of $\cosh \left(\eta-\eta_{*}\right)$ and $\sinh \left(\eta-\eta_{*}\right)$ and some of their mutual products for odd $d$, and as similar (not identical) polynomials plus a term $\pi\left(\eta-\eta_{*}\right)$ (with $\pi$ a constant) for even $d$. In the Lorentzian framework, the polynomials are similarly given in terms of circular rather than hyperbolic functions. Then we see that the above conclusion for $d=5$ can be extended for any arbitrary dimension $d$.

\section{STABILITY OF ASYMPTOTICALLY ANTI-DE SITTER WORMHOLES}

We shall show now that the above kind of instability problem is no longer present in the case where we use solution (2.2). When we express such a solution in terms of the conformal radial extra coordinate

$$
\begin{aligned}
\beta^{1 / 4} \eta= & \beta^{1 / 4} \eta_{*} \\
& +F\left[\arcsin \sqrt{\frac{\sqrt{\beta}\left(\cosh \left(2 \sqrt{\Lambda_{5}} r\right)-1\right)}{\sqrt{\beta} \cosh \left(2 \sqrt{\Lambda_{5}} r\right)-1}}, R(\beta)\right],
\end{aligned}
$$

with

$$
R(\beta)=\sqrt{\frac{\sqrt{\beta}+1}{2 \sqrt{\beta}}}
$$

and where again $\eta_{*}$ is an integration constant and $F$ is the elliptic integral of the first class [7], the scale factor can be written as

$$
a(\eta)=\gamma \operatorname{nc}\left(\beta^{1 / 4} \eta \mid m\right)
$$

in which we have absorbed the constant $\eta_{*}$ into $\eta$,

$$
\gamma=\frac{\sqrt{\beta}-1}{2 \Lambda_{5}}
$$

where $\operatorname{nc}(x \mid m)$ is an elliptic function with parameter $m$ [7]. Then, denoting $x=\beta^{1 / 4} \eta$, we have for the Euclidean differential equation for the coefficient $\nu$,

$$
\nu^{\prime \prime}+3 \beta^{1 / 4} \frac{\operatorname{sn}(x \mid m) \operatorname{dn}(x \mid m)}{\operatorname{cn}(x \mid m)} \nu^{\prime}=\ell(\ell+3) \nu,
$$

with sn, dn, and cn being elliptic functions as well [7]. Now, for the zero mode $\ell=0$ we obtain the analytical solution

$$
\begin{aligned}
\nu= & \nu_{0}+\frac{\nu_{0}^{\prime}}{2 m \beta^{1 / 4}}\{\operatorname{sn}(x \mid m) \operatorname{dn}(x \mid m) \\
& \left.-\frac{m_{1}}{\sqrt{m}} \arcsin (\sqrt{m} \operatorname{sn}(x \mid m))\right\},
\end{aligned}
$$


with $m_{1}=1-m$. It follows that though $\nu$ oscillates along the entire direction $\eta$, it does not diverge anywhere on that direction, so that, contrary to what happens for solution (2.1), the five-dimensional bulk for solution (2.2) appears to be stable to these zero-mode tensorial perturbations. It is worth noting, moreover, that in the Lorentzian case where the solution is given in terms of the elliptic function $\mathrm{cn}=1 / \mathrm{nc}$,

$$
a(\eta)=\gamma \operatorname{cn}\left(\beta^{1 / 4} \eta \mid m_{1}\right),
$$

the solution for the zero mode for coefficient $\nu$ is the same as in Eq. (3.5), but with parameter $m$ replaced for parameter $m_{1}$ and vice versa. In this way, though the $\ell=0$ gravitationalwave mode is not damped, neither does it increase with Lorentzian time $\eta$.

For $\ell \neq 0$ the differential equation for $\nu$ can be rewritten as

$$
\left[\operatorname{nc}(x \mid m) \nu^{\prime}\right]^{\prime}=\ell(\ell+3) \mathrm{nc}^{3}(x \mid m) \nu
$$

in the Euclidean description and as

$$
\left[\operatorname{cn}\left(x \mid m_{1}\right) \nu^{\prime}\right]^{\prime}=\ell(\ell+3) \mathrm{cn}^{3}\left(x \mid m_{1}\right) \nu
$$

in the Lorentzian description, with $x=\beta^{1 / 4} \eta$ in both cases. We have been unable to find an analytical solution in closed form for these differential equations, so that we will consider the limiting behaviors of $\nu$ as $\beta^{1 / 4} \eta \rightarrow 0,2 K, \ldots$ and $\beta^{1 / 4} \eta$ $\rightarrow K, 3 K, \ldots$, where $K$ is the complete elliptic integral [8]. In the former case for the Euclidean solution one can approximate $\nu$ to be given by

$$
\nu \simeq \nu_{0} \exp [\sqrt{\ell(\ell+3)} \eta],
$$

and in the latter Euclidean case $\nu$ tends to generally finite constant values. Thus, at least for $\eta$ intervals running up to finite numbers of complete elliptic integrals, we see that there is no instability arising from these $\ell \neq 0$ modes. For the Lorentzian description near $\beta^{1 / 4} \eta \rightarrow 0,2 K, \ldots$, if we choose for the constant $A^{2}$ the particular value $A^{2}=175 /\left(324 \Lambda_{5}\right)$, we obtain a solution in terms of the ultraspherical Gegenbauer polynomials [8],

$$
\nu \propto C_{\ell}^{3 / 2}(\eta),
$$

which tends either to vanish for odd $\ell$ or to a finite nonzero constant

$$
\nu=(-1)^{\ell / 2} \frac{\Gamma\left(\frac{3}{2}+\frac{\ell}{2}\right)}{\Gamma\left(\frac{3}{2}\right)\left(\frac{3}{2}\right)},
$$

for even $\ell$, as $\eta$ goes into these limiting values. For $\beta^{1 / 4} \eta$ $\rightarrow K, 3 K, \ldots$, the Lorentzian solution would again tend to a generally finite constant. Therefore, gravitational-wave perturbations do not induce any instability in the fivedimensional bulk for our nonsingular solution.
It is rather interesting to realize that the stability to tensorial perturbations of formally the same solutions for the scale factor will critically depend on the number of dimensions of the spacetime we deal with. In fact, there exist fourdimensional asymptotically anti-de Sitter Euclidean wormholes $[9,10]$ which are characterized by a Robertson-Walker metric and a scale factor which is exactly the same as that given by Eq. (2.2) for a cosmological constant $\Lambda_{4} \equiv \Lambda$. Such wormholes are usually obtained as the Euclidean solution that corresponds to the case of a massless scalar field which is conformally coupled to the Hilbert-Einstein gravity plus a cosmological constant $\Lambda[9,10]$. However, even for the case $\ell=0$, the solution appears to be unstable to the same kind of tensorial perturbations to which solution (2.2) is in fact stable. In this case, the differential equation is modified to be

$$
\sigma^{\prime \prime}+2 \beta^{1 / 4} \frac{\operatorname{sn}(x \mid m) \operatorname{dn}(x \mid m)}{\operatorname{cn}(x \mid m)} \sigma^{\prime}=0 .
$$

The solution to Eq. (3.10) is

$$
\sigma=\sigma_{0}+\frac{\sigma_{0}^{\prime}}{\beta^{1 / 4} m}\left\{E\left[A\left(\beta^{1 / 4} \eta\right), m\right]-m_{1} \beta^{1 / 4} \eta\right\}
$$

where $E$ is the elliptic integral of the second kind [8], and $A$ is the amplitude of the corresponding elliptic function [8]. We can readily check that in fact this solution diverges as $\eta \rightarrow \infty$. The counterpart for the Lorentzian baby universe will nevertheless be stable to gravitational-wave perturbations and corresponds to the solution

$$
\sigma=\sigma_{0}+\frac{\sigma_{0}^{\prime}}{\beta^{1 / 4} m_{1}}\left\{E\left[A\left(\beta^{1 / 4} \eta_{L}\right), m_{1}\right]-m \beta^{1 / 4} \eta_{L}\right\},
$$

with $\eta_{L}$ a compact time coordinate.

\section{CONCLUSIONS}

The conclusions of this paper are that, whereas the Garriga-Sasaki instanton based on a trivial extension to five or arbitrarily higher dimensions than the usual fourdimensional anti-de Sitter instanton and the kind of fourdimensional Euclidean asymptotically anti-de Sitter wormhole we have just discussed are unstable to the tensorial gravitational-wave-like perturbations, the baby universe associated with that wormhole and the nonsingular fivedimensional instanton which was used in [2] to replace the Garriga-Sasaki solution [1] are both stable to the Lorentzian counterpart of such perturbations. A caveat to these conclusions should be mentioned. It is that, since the tensorial instability of the five-dimensional anti-de Sitter instanton takes place as $r \rightarrow 0$, this instability would coincide with the other kind of instability identified for that solution which arises from the invariant Weyl tensor $C^{2}$ also at $r=0$. Whether or not these two apparently distinct kinds of instabilities share a common ultimate origin is to be investigated. We note, moreover, that the presence of such instabilities 
appears to be independent of the value of $r$ at which the brane is placed. On the other hand, the five-dimensional wormhole instanton, and hence the brane constructed from it, is stable as one approaches the limiting values of $r$ at both $r=0$ and $r=\infty$.

\section{ACKNOWLEDGMENTS}

The author thanks Mariam Bouhmadi and Carmen L. Sigüenza for useful discussions. This work was supported by DGICYT under Research Project BMF2002-03758.
[1] J. Garriga and M. Sasaki, Phys. Rev. D 62, 043523 (2000); K. Koyama and J. Soda, Phys. Lett. B 483, 432 (2000); L. Anchordoqui and K. Olsen, Mod. Phys. Lett. A 16, 1157 (2001); L. Anchordoqui, C. Nuñez, and K. Olsen, J. High Energy Phys. 10, 050 (2000); H.A. Chamblin and A.S. Reall, Nucl. Phys. B562, 133 (1999); S. Kanno, M. Sasaki, and J. Soda, Prog. Theor. Phys. 109, 357 (2003); R. Brandenberger, G. Geshnizjani, and S. Watson, Phys. Rev. D 67, 123510 (2003).

[2] M. Bouhmadi and P.F. González-Díaz, Phys. Rev. D 65, 063510 (2002); M. Bouhmadi-López, P.F. González-Díaz, and A. Zhuk, Class. Quantum Grav. 19, 4863 (2002); P.F. González-Díaz, Nucl. Phys. B619, 646 (2001).

[3] P. Binétruy, C. Deffayet, and D. Langlois, Nucl. Phys. B565, 269 (2000); P. Binétruy, C. Deffayet, U. Ellwanger, and D.
Langlois, Phys. Lett. B 477, 285 (2000); P.F. González-Díaz, ibid. 481, 353 (2000).

[4] P. Ginsparg and M.J. Perry, Nucl. Phys. B222, 245 (1983).

[5] E.M. Lifshitz, J. Phys. (Moscow) 10, 116 (1946); E.M. Lifshitz and I.M. Khalatnikov, Adv. Phys. 12, 185 (1963).

[6] P.F. González-Díaz, Phys. Rev. D 36, 3651 (1987).

[7] P.F. González-Díaz, Phys. Lett. B 512, 127 (2001).

[8] M. Abramowitz and I. Stegun, Handbook of Mathematical Functions (Dover, New York, 1964).

[9] P.F. González-Díaz, "Elliptic and Circular Wormholes," gr-qc/9306031.

[10] C. Barceló, L.J. Garay, P.F. González-Díaz, and G.A. Mena Marugán, Phys. Rev. D 53, 3162 (1996). 